\title{
Thermoelectric Power of Cu-Zn Ferrites
}

\author{
Hussain Abd-Elkariem Dawoud
}

Physics Department, Islamic University of Gaza, Gaza Strip, Palestine.

Email: hdawoud@iugaza.edu.ps

Received May 24 $4^{\text {th }}, 2011$; revised August $24^{\text {th }}, 2011$; accepted October $4^{\text {th }}, 2011$.

\begin{abstract}
A series of $\mathrm{Cu}$-Zn mixed ferrites with composition formula $\mathrm{Cu}_{1-x} \mathrm{Zn}_{x} \mathrm{Fe}_{2} \mathrm{O}_{4}$ is prepared by the double sintering ceramic technique. Thermoelectric power studies are performed over a temperature range of 300 to $800 k$ by a deferential method. The results showed a negative value for the Seebeck coefficient $S$ for all samples, and all compositions exhibited an n-type semiconductors behavior in the measured range of temperature. The values of charge carrier concentration $n$ and the Fermi energy were determined. The values of $n$ were found to decrease as temperature increased, while Fermi energy directed to more negative values when Zn content is increased. On the basis of these results a mechanism for the conduction in Cu-Zn ferrites is suggested and the properties of the mention compounds were determined.
\end{abstract}

Keywords: Ferrites, Thermoelectric Power, Fermi Energy, Seebeck Coefficient

\section{Introduction}

Spinel ferrites are commercially important materials because of their excellent magnetic and electrical properties. Interesting physical and chemical properties of the magnetically diluted ferrites arise from the ability of these compounds to distribute the cations amongst the available tetrahedral A- and octahedral B-sites. Ferrites are able to fulfill a wide range of applications from micro wave to radio frequencies, are of great importance from both fundamental and applied research points of view $[1,2]$. Because Zinc is non-magnetic divalent ions that occupy essentially tetrahedral A sites, when substituted in ferrites [3]. Gonchar and Andreev [4] have reported that $\mathrm{Ni}-\mathrm{Cu}-\mathrm{Zn}$ ferrites with less content of $\mathrm{Zn}$ could obtain high Curie temperature, but the initial permeability of Ni-Cu-Zn ferrites reached. The substitution of $\mathrm{Cu}$ brings about a structural phase transition accompanied by the reduction in the crystal symmetry due to co-operative John-Teller effect $[5,6]$, which ultimately results in some interesting electrical and magnetic properties. Various investigations $[7,8]$ studied the electrical and thermal power of the spinel ferrites and have found that they have semi-conducting properties of $\mathrm{n}$ or $\mathrm{p}$-type. In this article, the electric and thermoelectric power of $\mathrm{Cu}-\mathrm{Zn}$ ferrites have been studied experimentally as a function of composition and temperature in order to understand the conduction mechanism in these samples.

\section{Experimental Technique}

The polycrystalline Zinc-substituted Copper ferrites having compositional formula $\mathrm{Cu}_{1-\mathrm{x}} \mathrm{Zn}_{\mathrm{x}} \mathrm{Fe}_{2} \mathrm{O}_{4}$ where $\mathrm{x}$ stepped $0 \leq 0.2 \geq 1$, were prepared by the double sintering ceramic technique [9], from pure oxides powders $\mathrm{Fe}_{2} \mathrm{O}_{3}$, $\mathrm{CuO}$ and $\mathrm{ZnO}$. Oxides were mixed in stoichiometric proportions and prefired at $750^{\circ} \mathrm{C}$ for $5 \mathrm{~h}$. The prefired powders were grounded and compressed at constant pressure $\left(3 \times 10^{8} \mathrm{~Pa}\right)$ in the form of disk shape of $13 \mathrm{~mm}$ diameter. The samples were finally sintered at $1150^{\circ} \mathrm{C}$ for 5 hours and then slowly cooled to room temperature with cooling rate of $2^{\circ} \mathrm{C} / \mathrm{min}$.

The electrical measurements were performed by twoprobe method; silver past was applied on both sides of the samples to make good ohmic contact.

Thermoelectric power studies were carried out as a function of composition and temperature by differential method [10]. The sample holder for measuring the Seebeck coefficient consists of two non-magnetic copper electrodes between which the sample is fixed. An auxiliary heating coil is fixed to upper electrode for additional heating to maintain a temperature difference of about 15 degree between the two faces of the sample. A temperature of both surfaces of the sample was measured by the same type of two thermocouples. The Seebeck coefficient $\mathrm{S}$ was calculated using the relation

$$
\mathrm{S}=\Delta \mathrm{E} / \Delta T
$$

where $\Delta \mathrm{E}$ is the thermoelectric potential difference pro- 
duced across the sample due to the temperature difference $\Delta T$.

\section{Results and Discussion}

The variation values of Seebeck coefficient with temperature for each sample of Cupper zinc ferrite having a compositional formula $\mathrm{Cu}_{1-\mathrm{x}} \mathrm{Zn}_{\mathrm{x}} \mathrm{Fe}_{2} \mathrm{O}_{4}$ where $\mathrm{x}$ stopped 0 $\leq 0.2 \geq 1$, have bean calculated from the experimental

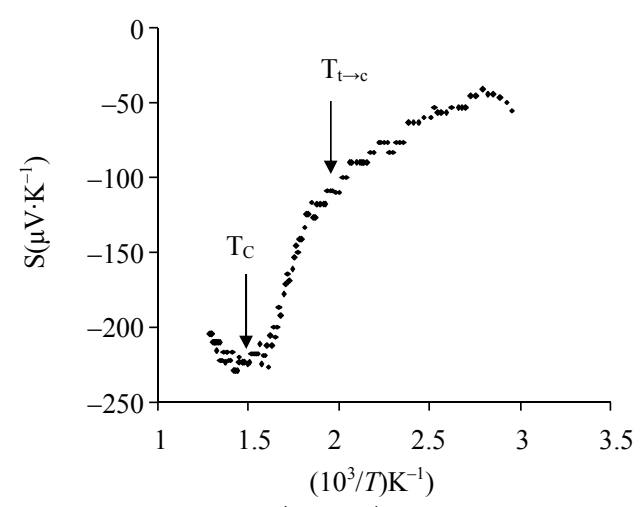

(a)

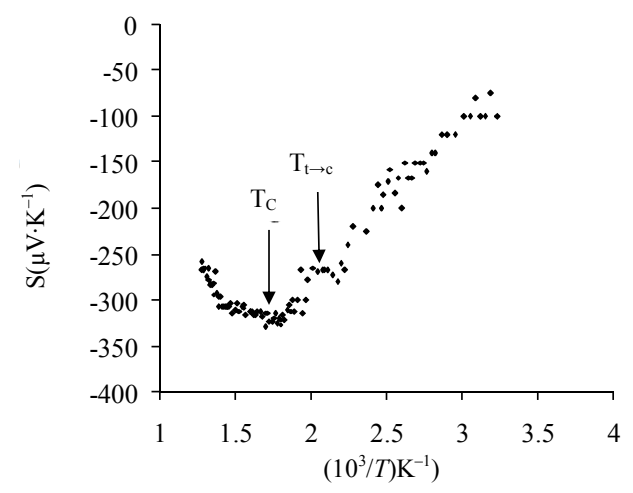

(c)

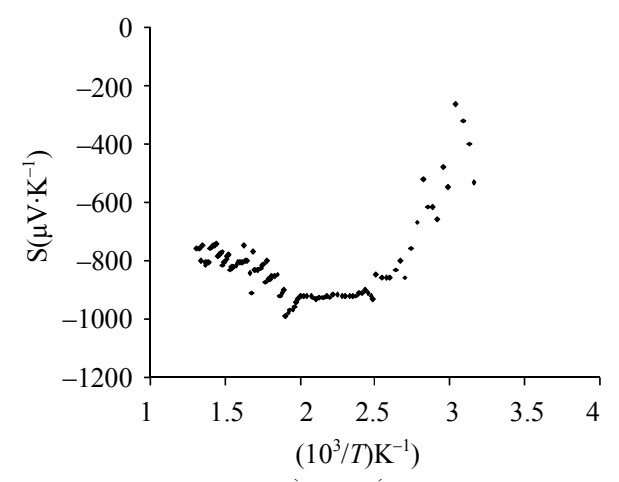

(e) values of thermoelectric motive force, and depicted against the temperature as shown in Figures 1(a)-(f). It is noticed that the sign of the Seebeck coefficient for all samples is negative, indicting that the $\mathrm{Cu}-\mathrm{Zn}$ ferrite behave as $\mathrm{n}$ type semiconductor. The conduction mechanism in these ferrites is due to electrons [11], and many authors obtained similar results $[12,13]$.

Several features can be obtained from Figures 1(a)-(f),

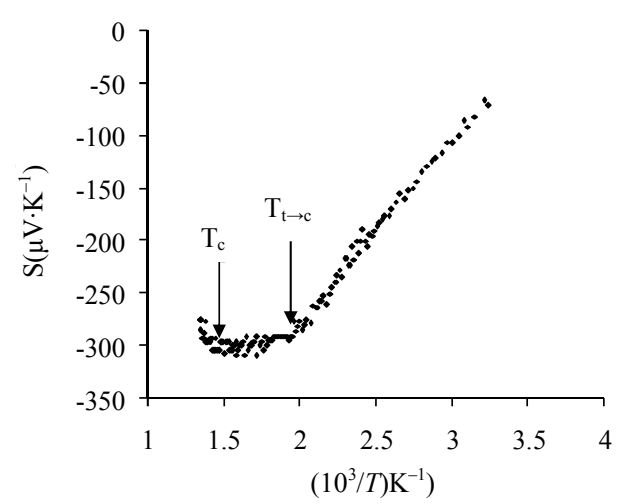

(b)

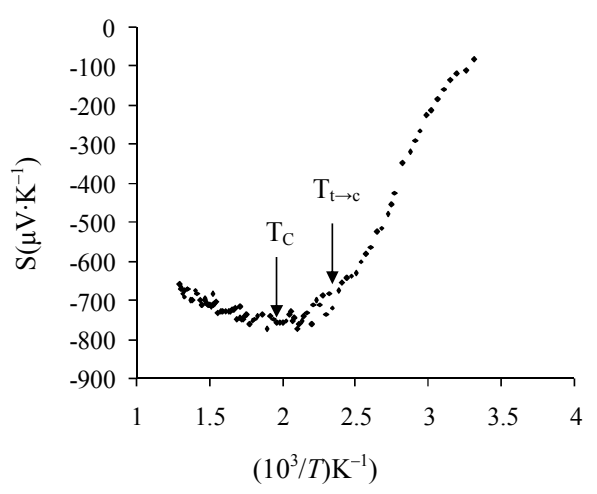

(d)

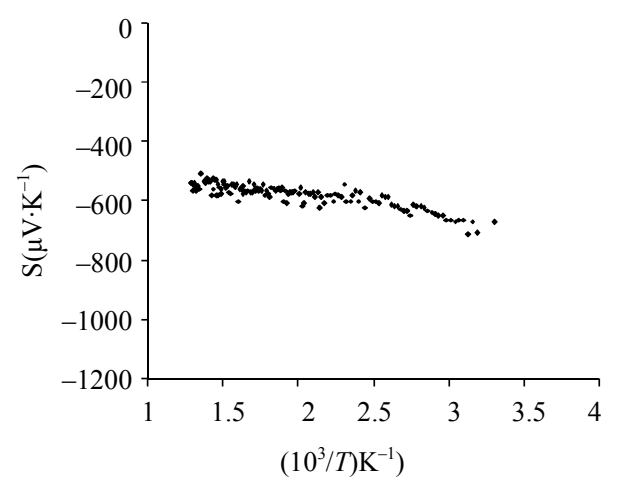

(f)

Figure 1. (a) The variation of Seebeck coefficient (S) with $10^{3} / T$ (for sample of $x=0.0$ ); (b) The variation of Seebeck coefficient (S) with $10^{3} / T$ (for sample of $x=0.2$ ); (c) The variation of Seebeck coefficient (S) with $10^{3} / T$ (for sample of $x=0.4$ ); (d) The variation of Seebeck coefficient (S) with $10^{3} / T$ (for sample of $x=0.6$ ); (e) The variation of Seebeck coefficient (S) with $10^{3} / T$ (for sample of $x=0.8$ ); (f) The variation of Seebeck coefficient (S) with $10^{3} / T$ (for sample of $x=1.0$ ). 
compositions of $x \leq 0.6$ have two change of slope in $S$ against temperature, the first change at lower temperature can referred to structural change from tetragonal to cubic system. The transition from tetragonal to cubic $T_{t \rightarrow c}$ was found to decrease with increasing $\mathrm{Zn}$ content as shown in Figure 2. In other words the tetragonality is in agreement with the results of [14].

At higher temperature Seebeck coefficient $\mathrm{S}$ decrease in negativity with increasing temperature, up to a particular temperature, hereafter referred to as Seebeck coefficient transition temperature $T_{\mathrm{c}}$. However, by further increase of temperature, the values are found to decrease in negativity. The minimum values observed in Seebeck coefficient may be attributed to a magnetic transition, where the ferromagnetic material becomes paramagnetic. On the other hand, in the case of samples of $x=\geq 0.8$, the Seebeck coefficient is found to be increased slightly as the temperature increased unlike other samples, and there is no transition $T_{c}$ was found.

This is due to the fact that these samples having higher Zinc concentration with weak tetrahedral A-site and octahedral B-site super exchange interaction. This behavior is obvious because both samples have higher concentration of Zinc with paramagnetic structure at room temperature [14]. Similarly, they behave like normal solid with slightly increase in Seebeck coefficient values with temperature. However, the change of slope could not be observed in the case of the two samples i.e. of $\mathrm{x}=\geq 0.8$ since they are diamagnetic at room temperature [15].

Generally, the change of slope for samples of $\mathrm{x} \leq 0.6$ is attributed to change in conductivity mechanism. The conduction at lower temperature (below Curie temperature) is due to hopping of electrons [12] between $\mathrm{Fe}^{3+}$ and $\mathrm{Fe}^{2+}$ ions, whereas at higher temperature (above Curie temperature) due to polaron hopping [15].

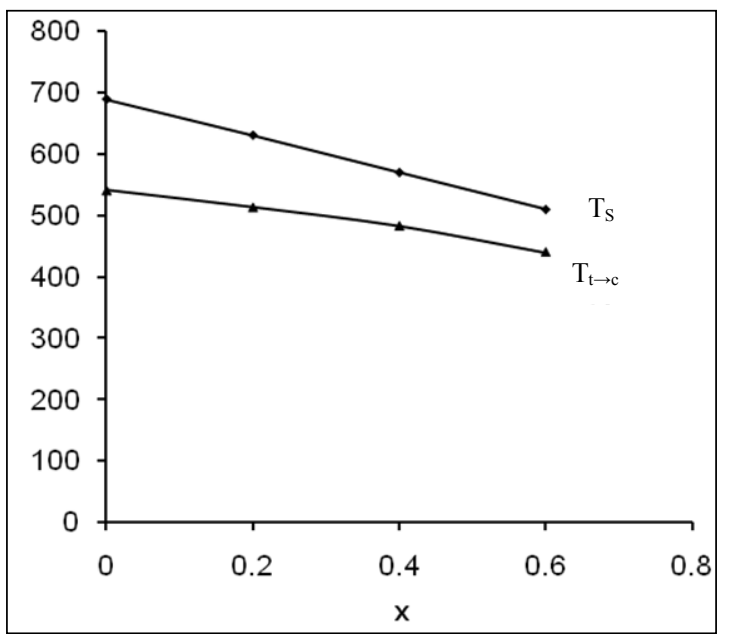

Figure 2. The variation of $T_{t \rightarrow c}$ and $T_{S}$ with composition $x$.
Following according to Wu et al. [16] the Seebeck coefficient $\mathrm{S}$ is expressed in terms of $\mathrm{Fe}^{3+}$ and $\mathrm{Fe}^{2+}$ ions, considering the small polaron hopping conduction mechanism as follows:

$$
\mathrm{S}=-(k / e) \ln \left\{\beta\left[\mathrm{Fe}^{3+}\right]_{\mathrm{B}} /\left[\mathrm{Fe}^{2+}\right]_{\mathrm{B}}\right\}
$$

where $\left[\mathrm{Fe}^{3+}\right]_{\mathrm{B}}$ and $\left[\mathrm{Fe}^{2+}\right]_{\mathrm{B}}$ are the concentration of the $\mathrm{Fe}^{3+}$ and $\mathrm{Fe}^{2+}$ ions in the octahedral sites, respectively and $\beta=1$. Dawoud et al. [17] reported that the increase of $\mathrm{Zn}$ substitution tends to increase the $\mathrm{Fe}^{2+}$ ions as demon-strated in above equation.

In addition, the increase of $\mathrm{Zn}$ content is associated with a decrease in copper content. In turn the possibility of $\mathrm{Cu}^{+}$formation and $\mathrm{Cu}^{2+} \leftrightarrow \mathrm{Cu}^{1+}$ hopping process and the number of holes involved will be reduced. As a result $\mathrm{S}$ becomes more negative with the increase of $\mathrm{x}$ (Zinc content).

\section{Charge Carrier Concentration (n)}

In the case of low mobility semiconductor like ferrites having exceedingly levels, the value of $\mathrm{N}$, the density of state can be taken as $10^{22} \mathrm{~cm}^{-3}[18]$.

$$
\mathrm{n}=N / V[N / 1+\exp (-S e / k)]
$$

The value of charge carriers concentration/unit volume have been calculated for all the samples by using the values of the Seebeck coefficient $S$, and the relation between $\mathrm{n}$ and temperature is depicted in Figures 3(a)-(c) for samples of $x=0.0,0.2$ and 0.4 respectively. The values of $\mathrm{n}$ for all samples are also listed in Table 1. The figures illustrated that, the values of $\mathrm{n}$ decreased with the increasing the temperature.

\section{Fermi Energy (EF)}

The thermoelectric power data were utilized to determine the Fermi energy. The Fermi energy of $\mathrm{Cu}-\mathrm{Zn}$ ferrite was calculated employing the following expression $[10,19]$.

$$
\mathrm{E}_{\mathrm{F}}=\mathrm{eST}-\mathrm{AKT}
$$

where $\mathrm{K}, \mathrm{e}, \mathrm{S}$ and $\mathrm{T}$ are Boltzmann constant, electronic charge, Seebeck coefficient and absolute temperature respectively. A is a dimensionless constant related to the kinetic energy of the charge carrier, and has values of 0 or 2. Two series of values of $E_{F}$ were obtained, over the temperature range from $300 \mathrm{~K}$ up to $800 \mathrm{~K}$, for $\mathrm{A}=0$ and $A=2$. Figure 4(a)-(c) represent the variation of Fermi energy with temperature. The extrapolation of the linear part of the two series of $\mathrm{E}_{\mathrm{F}}$, for $\mathrm{A}=0$ and $\mathrm{A}=2$ intersects at $\mathrm{T}=0$.

The corresponding value of $E_{F}$, the point of interception at ordinate, gives the Fermi energy at zero tempera- 


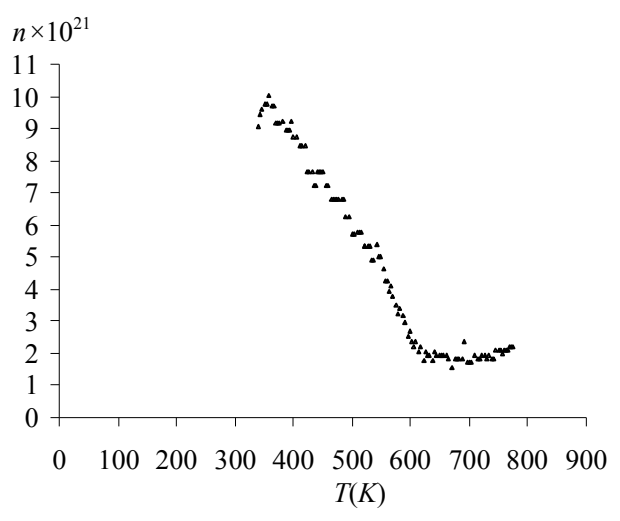

(a)

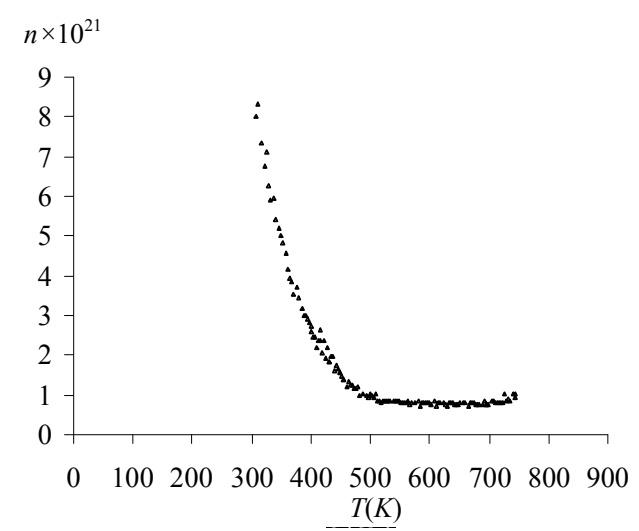

(b)

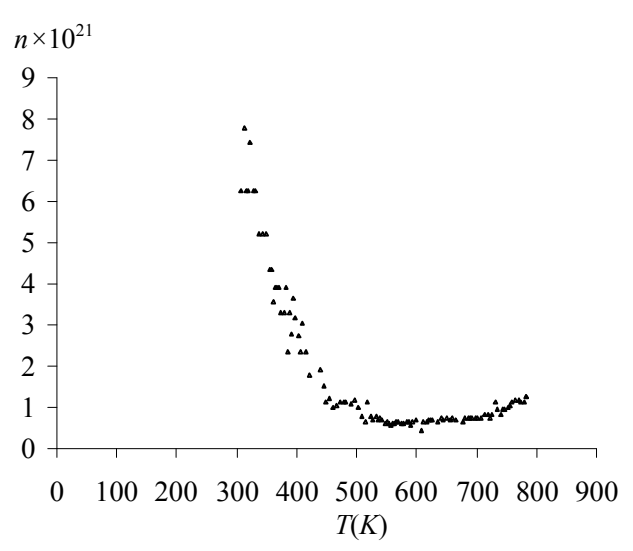

(c)

Figure 3. (a) Temperature dependence of charge carrier $n$ for sample of $x=0.0$; (b) Temperature dependence of charge carrier $n$ for sample of $x=0.2$; (c) Temperature dependence of charge carrier $n$ for sample of $x=0.4$.

ture $\mathrm{E}_{\mathrm{F}}(0)$; and Figures 4(a)-(c) indicates the variation of Fermi energy with temperature. Also Table 1 lists the values of Fermi energy obtained for each composition. It is noticed that $\mathrm{E}_{\mathrm{F}}(0)$ is positive for sample of $\mathrm{x}=0$ and sample of $\mathrm{x}=0.2$ then it decreases to the negative value i.e. at the left hand side of the gape, as zinc ion increased.
Table 1. The values of Seebeck coefficient, Curie temperature transition temperarure from tetragonal to cubic $T_{t \rightarrow c}$ and Fermi energy for $\mathrm{Cu}-\mathrm{Zn}$ ferrites.

\begin{tabular}{ccccc}
\hline $\mathrm{x}$ & $\mathrm{S}$ at $374 \mathrm{~K}$ & $\mathrm{~T}_{\mathrm{S}}(\mathrm{K})$ & $\mathrm{T}_{\mathrm{t} \rightarrow \mathrm{c}}(\mathrm{K})$ & $\mathrm{E}_{\mathrm{f}}(\mathrm{ev})$ \\
\hline 0.0 & -53.8 & 688 & 540 & $2.4 \times 10^{-20}$ \\
0.2 & -158 & 630 & 512 & $1.6 \times 10^{-20}$ \\
0.4 & -166.7 & 569 & 482 & $0.4 \times 10^{-20}$ \\
0.6 & -515 & 509 & 439 & $-0.6 \times 10^{-20}$ \\
0.8 & -800 & - & - & $-0.8 \times 10^{-20}$ \\
1.0 & -636.7 & - & - & $-1.0 \times 10^{-20}$ \\
\hline
\end{tabular}

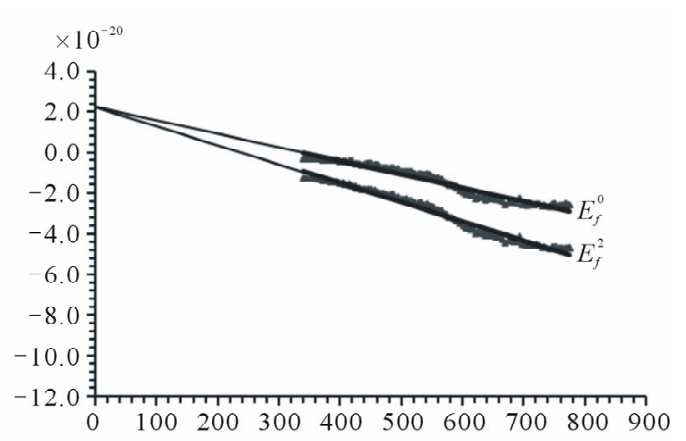

(a)

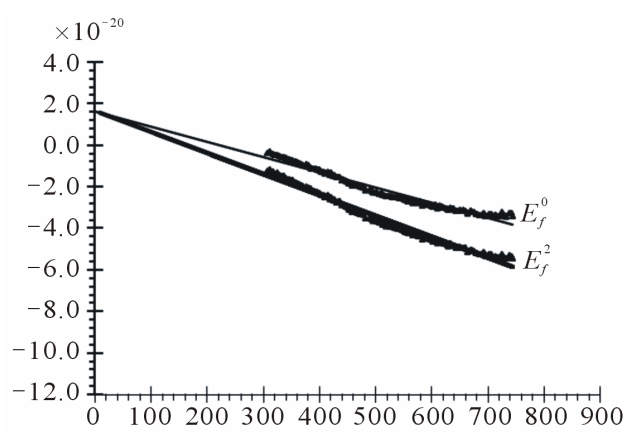

(b)

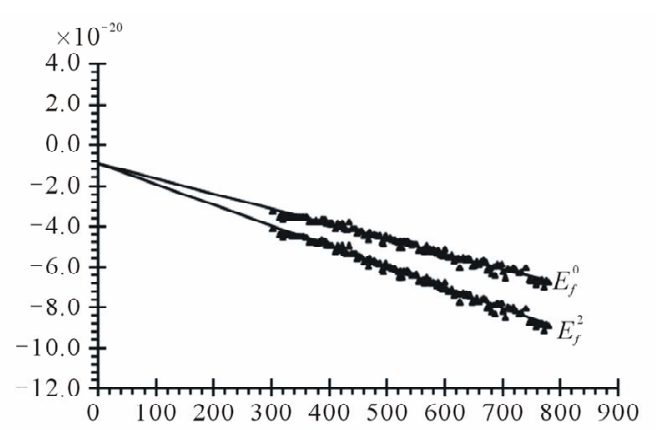

(b)

Figure 4. (a) Plot of $E_{f}$ against temperature for sample of $x$ $=0.0$; (b) Plot of $E_{f}$ against temperature for sample of $\mathbf{x}=$ 0.2 ; (c) Plot of $E_{f}$ against temperature for sample of $x=1.0$. 
Mazen et al. [10] reported that for $\mathrm{Cu}$ ferrite, $\mathrm{E}_{\mathrm{F}}(0)$ is positive at $\mathrm{T}=0 \mathrm{~K}$. Patil et al. [11] reported that when heating $\mathrm{CuFe}_{2} \mathrm{O}_{4}$ sample, two temperature domains are noted in which the concentration of $\mathrm{B}$-site $\mathrm{Cu}^{2+}$ ions is increased and the interstice cation exchange is given as

$$
\mathrm{Cu}_{\mathrm{A}}^{2+}+\mathrm{Fe}_{\mathrm{B}}^{3+} \rightarrow \mathrm{Cu}_{\mathrm{B}}^{2+}+\mathrm{Fe}_{\mathrm{A}}^{3+}
$$

This reaction gives n-type condition, while at low temperature the conduction process for $\mathrm{CuFe}_{2} \mathrm{O}_{4}$ at lower temperature is

$$
\mathrm{Cu}_{\mathrm{A}}^{1+}+\mathrm{Cu}_{\mathrm{A}}^{2+} \rightarrow \mathrm{Cu}_{\mathrm{A}}^{2+}+\mathrm{Cu}_{\mathrm{A}}^{1+}
$$

This gives only p-type conduction depending on the relative concentration of $\mathrm{Cu}^{1+}$ of $\mathrm{Cu}^{2+}$ ions on A-sites. For the present system Fermi energy is positive for $\mathrm{Cu}$ ferrite and reaction (2) is fevered. While as the Zinc content is increased, Fermi energy goes to negative and reaction (1) is fevered since the increasing of the Zinc content tend to decrease $\mathrm{Cu}$ content at $\mathrm{A}$-site this tend to increase the negativity of Fermi energy at absolute zero.

\section{Conclusions}

1) The $\mathrm{Cu}-\mathrm{Zn}$ ferrite behaves as n-type semiconductor.

2) The transition from tetragonal structure to cubic was found to decrease with increasing of $\mathrm{Zn}$ content.

3) The minimum values observed in Seebeck coefficient may be attributed to a magnetic transition, where the ferromagnetic material becomes paramagnetic.

4) The conduction at lower temperature (below Curie temperature) is due to hopping of electrons between $\mathrm{Fe}^{3+}$ and $\mathrm{Fe}^{2+}$ ions, whereas at higher temperature (above $\mathrm{Cu}-$ rie temperature) due to polaron hopping.

5) For the present system Fermi energy is positive for $\mathrm{Cu}$ ferrite while as the Zinc content is increased, Fermi energy goes to negative value.

\section{REFERENCES}

[1] P. K. Roy and J. Bera, "Enhancement of the Magnetic Properties of Ni-Cu-Zn Ferrites with the Substitution of a Small Fraction of Lanthanum for Iron," Materials Research Bulletin, Vol. 42, No. 1, 2007, pp. 77-83. doi:10.1016/j.materresbull.2006.05.009

[2] I. Z. Rahman and T. T. Ahemed, "A Study on Cu Substituted Chemically Processed Ni-Zn-Cu Ferrites," Journal of Magnetism and Magnetic Materials, Vol. 290-291, 2005, pp. 1576-1579. doi:10.1016/i.jmmm.2004.11.250

[3] D. Ravinder, "Thermoelectric Power and Electric Conductivity of Cd-Substituted Copper Ferrite," Materials Letters, Vol. 44, No. 3-4, 2000, pp. 130-138. doi:10.1016/S0167-577X(00)00015-X

[4] A. Gonchar, V. Andreev, L. Letyuk, A. Shishkanov and V. Maiorov, "Problems of Increasing of Thermostability of Highly Permeable Ni-Zn Ferrites and Relative Materi- als for Telecommunications," Journal of Magnetism and Magnetic Materials, Vol. 254-255, 2003, pp. 544-546. doi:10.1016/S0304-8853(02)00860-0

[5] M. C. Dimri, A. K. Verma, S. C. Kashyap, D. C. Dube and O. P. Thakur, "Structural, Dielectric and Magnetic Properties of NiCuZn Ferrite Grown by Citrate Precursor Method," Materials Science and Engineering: B, Vol. 133, No. 1-3, 2006, pp. 42-48. doi:10.1016/j.mseb.2006.04.043

[6] P. A. Jadhav, R. S. Devan, Y. D. Kolekar and B. K. Chougule, "Structural, Electrical and Magnetic Characterizations of Ni-Cu-Zn Ferrite Synthesized by Citrate Precursor Method," Journal of Physics and Chemistry of Solids, Vol. 70, No. 2, 2009, pp. 396-400. doi:10.1016/j.jpcs.2008.11.019

[7] D. Ravinder, "Thermoelectric Power Studies of Zinc Substituted Copper Ferrite," Journal of Alloys and Compounds, Vol. 291, No. 1-2, 2000, pp. 208-214. doi:10.1016/S0925-8388(99)00287-X

[8] K. V. Kumar and D. Ravinder, "Electrical Transport Properties of Erbium Substituted Ni-Zn Ferrite," International Journal of Inorganic Materials, Vol. 3, No. 7, 2001, pp. 661-666. doi:10.1016/S1466-6049(01)00194-5

[9] S. A. Mazen, "Electrical Conductivity and Thermoelectric Power of Cu-Ti Ferrite," Materials Chemistry and Physics, Vol. 56, No. 2, 1998, pp. 102-107. doi:10.1016/S0254-0584(98)00136-9

[10] S. A. Mazen and A. Elfalaky, "Thermoelectric Power and Electrical Conductivity of Cu-Ti Ferrite," Journal of Magnetism and Magnetic Materials, Vol. 195, No. 1, 1999, pp. 148-155. doi:10.1016/S0304-8853(98)00348-5

[11] B. L. Patil, S. R. Sawant and S. A. Patil, "Electrical and Magnetic Studies of $\mathrm{Ba}_{3} \mathrm{Co}_{2} \mathrm{Fe}_{23-12 x} \mathrm{Mn}_{12 \mathrm{x}} \mathrm{O}_{4}$ Hexaferrites Type," Physical State Solid (A), Vol. 133, No. 1-2, 1992, p. 147.

[12] P. V. Reddy, V. D. Reddy and D. Ravinder, "Thermopower Studies of Lithium-Zinc Mixed Ferrites," Physical State Solid (A), Vol. 127, No. 2, 1991, pp. 439-450.

[13] R. Manjula, V. R. K. Murthy and J. Sobhandri, "Electric Conduction in Ni-Zn Ferrite," Journal of Applied Physics, Vol. 59, No. 5, 1986, p. 2929.

[14] H. A. Dawoud and S. K. K. Shaat, "Initial Permeability and Conductivity of $\mathrm{Cu}-\mathrm{Zn}$ Ferrite," Islamic University Journal, Vol. 14, No. 1, 2006, pp. 165-182.

[15] C. N. Chinnasamy, A. Narayanasamy, N. Ponpandian and K. Chottopdhyay, "Size Dependent Magnetic Behavior of Noncrystalline Spinel Ferrite," Journal Materials Science and Engineering A, Vol. 304, 2001, p. 983. doi:10.1016/S0921-5093(00)01611-7

[16] C. C. Wu, S. Krishnan and T. O. Mason, "Thermopower Composition Dependence in Ferrospinel," Journal of Solid State Chemistry, Vol. 37, No. 2, 1981, pp. 144-150. doi:10.1016/0022-4596(81)90079-7

[17] H. M. Zaki and H. A. Dawoud, "Far Infrared Spectra for Cooper-Zinc Mixed Ferrite," Physica B: Condensed Matter, Vol. 405, No. 21, 2010, p. 4479. doi:10.1016/j.physb.2010.08.018 
[18] F. J. Morin, "Charge Carrier and Mobility of Cu-Ti Ferrite," Physical Review, Vol. 93, 1970, p. 433.

[19] A. J. Bosman and C. Crevecoevr, "Electrical Conduction in Li-Doped CoO," Journal of Physics and Chemistry of Solids, Vol. 30, No. 5, 1969, pp. 1151-1160.

doi:10.1016/0022-3697(69)90372-2 